\title{
A Self-management App for Maternal Mental Health
}

\author{
Dr Anne Moorhead \\ School of Communication \& Media \\ Ulster University \\ a.moorhead@ulster.ac.uk
}

\author{
Dr Raymond Bond \\ School of Computing \\ Ulster University \\ rb.bond@ulster.ac.uk
}

\author{
Professor Maurice Mulvenna \\ School of Computing \\ Ulster University \\ md.mulvenna@ulster.ac.uk
}

\author{
Professor Siobhan O'Neill \\ School of Psychology \\ Ulster University \\ sm.oneill@ulster.ac.uk
}

\author{
Nuala Murphy \\ Moment Health \\ Belfast \\ nuala@momenthealth.io
}

\begin{abstract}
Femtech (female technology) describes digital technologies focusing on women's health, which is gaining enormous growing global attention and investment. One such women's health area is maternal mental health, which is an increasing problem with a lack of accessible resources. The overall aim of this research programme is to provide evidence to inform, enhance and evaluate the effectiveness of the Moment Health App in order to improve maternal mental health among mothers and their families, and to make maternal mental health mainstream. A mobile application (app) has been developed for new and expectant mothers and their families, which screens for prenatal and postnatal depression and associated anxieties, and includes additional features such as a helpful guide to practical and accessible coping strategies. The app consists of easy-to-use tools including an early intervention symptoms checker, a mood tracker, and a location tool. Further research is ongoing to advance the app, and to test its' effectiveness in improving maternal mental health. This research programme is providing the evidence to support the stageof-the-art development, ongoing advances, and evaluations to make maternal mental health mainstream through the use of technology, and thus contributing to the growing femtech.
\end{abstract}

Keywords: Maternal mental health Mobile application Femtech

\section{INTRODUCTION}

Maternal mental health is an important and increasing problem. However, there is limited resources to monitor and provide support to mothers and their families in the area of maternal mental health. This work-in-progress paper outlines the problem of maternal mental health and one potential solution is digital in the form of a mobile application (known as Moment Health App), which has been designed and developed to promote maternal mental health among expectant and new mothers, and their families. This app screens for prenatal and postnatal depression and associated anxieties, and includes additional features such as a helpful guide to practical and accessible coping strategies. This research is in the area of femtech. An interdisciplinary team of researchers is collaborating with a technology company, Moment Health, Belfast, to inform the development of this app. The company is committed to making maternal mental health mainstream.

\subsection{Problem - Maternal Mental Health}

Pregnancy and the arrival of a newborn is a very exciting time in any family, it is also a time when emotions, both positive and negative, may impact on the health and well-being of the mother and family. The World Health Organisation [1] defined maternal mental health as "a state of well-being in which a mother realises her own abilities, can cope with the normal stresses of life, can work productively and fruitfully, and is able to make a contribution to her community." According to the World Health Organisation [2], worldwide there are approximately $10 \%$ of pregnant women and $13 \%$ of women who have just given birth experience a mental disorder, primarily depression. Within the UK, up to one in five women develop mental health problems during pregnancy or in the first year after childbirth [3]. Key findings from the recent Royal College of Obstetricians and Gynaecologists' survey of 2300 women revealed that $81 \%$ of women had experienced a maternal mental health problem [3]. The pain this causes women and their 
families, the negative impact on their health and wellbeing, and the economic costs to individuals, the NHS and the nation are considerable [4]. It's been estimated that maternal mental health problems cost the UK £8.1 billion each year [5].

Emotions are a strong feeling deriving from one's circumstances, mood, or relationships with others. The literature clearly states that it is normal for expectant mothers to have emotional changes due to hormones during pregnancy. In addition, emotions during pregnancy can impact upon decision making of the individual [6]. The six most reported types of emotions during pregnancy and birth are: surprise, joy, anger, fear, love, and sadness [6-8]. From these six emotions, half are negative and half are positive, thus indicating that pregnancy and birth can produce mixed feelings. However, the literature emphasises more on negative than positive emotions. This is particularly true for the emotions for mothers after giving birth. The negative emotions result in well reported baby blues, postnatal depression and the most severe, postpartum psychosis [9]. It is clear that emotions among mothers need to be monitored in order to improve maternal mental health.

\subsection{Need and Rationale}

The Royal College of Midwives [6] surveyed midwives and maternity support workers who reported that emotional support should be the main focus of postnatal care (midwives 61\% and maternity support workers $78 \%$ ). These figures were significantly higher than clinical observation (midwives 20\% and maternity support workers $13 \%$ ) and health promotion (midwives 19\% and maternity support workers $8 \%$ ). In total, $41 \%$ of midwives and $40 \%$ of maternity support workers reported that there is usually enough time and resources to support, and inform women on maternal emotional well-being. These results clearly indicate that there is not enough time and resources for maternal emotional wellbeing but it should be the main focus of postnatal care. Thus this is a clear message from the Royal College of Midwives that emotional support should be the main focus of postnatal care and there needs to be more staff time and resources employed in this area. This survey is further supported by more recent findings from the Maternal Mental Health Alliance [10] that in almost half of the UK, pregnant women and new mothers have no access to specialist community maternal mental health services.

NICE Guidelines [11] in the UK on antenatal and postnatal mental health quality standard (QS115) states that "women are asked about their emotional well-being at each routine antenatal and postnatal contact." The guideline states the quality measures, which includes evidence of arrangements for healthcare professionals to ask women about their emotional wellbeing at all routine antenatal and postnatal contacts. The stated outcomes include women's satisfaction with being able to discuss any concerns or worries at routine appointments, and identification of mental health problems. Although this guideline is clear, there are questions around the implementations and the available resources for its' effectiveness and success.

The literature clearly states that maternal mental health is an increasing problem but there is a lack of resources. Thus there is a need for more accessible resources for mothers and their families to enhance their mental health.

Femtech (female technology) which is a relatively new term to describe digital technologies focusing on women's health, which is gaining enormous growing global attention and investment. Femtech refers to software, diagnostics, products, and services that use technology to improve women's health. It involves the use of digital health to motivate patients to access and use applications for managing women's health issues [12]. This research is in this area of femtech, i.e. using digital technology to address maternal mental health.

The overall aim of this research programme is to provide evidence to inform, enhance and evaluate the effectiveness of Moment Health App in order to improve maternal mental health among mothers and their families, and to make maternal mental health mainstream. This app is leading on from previous work of the research team as health apps have been shown to improve users' access to services and health outcomes [13]. Apps use social media platforms for communication and collaboration purposes. Social media brings a new dimension to healthcare as it offers a medium to be used by the public, patients, and health professionals to communicate about health issues with the possibility of potentially improving health outcomes [14]. In addition, this app was developed in Northern Ireland, where there are additional concerns about the higher rates of mental illness and the risk of transgenerational transmission of trauma related mental illnesses associated with Northern Ireland's history of violence [15].

\section{DIGITAL SOLUTION - MOMENT HEALTH APP}

A potential solution to the increasing problem of maternal mental health and the lack of resources in this area is the development of a digital solution. This solution is in the form of a mobile application, referred to in this paper as the Moment Health App. 


\subsection{App Design}

This app has been developed with expertise from clinicians and healthcare professionals, and is aimed at new and expectant mothers and their families. It screens for prenatal and postnatal depression and associated anxieties, and includes additional features such as a helpful guide to practical and accessible coping strategies. When a parent or parent-to-be downloads the app, they will have access to several key, easy-to-use tools including an early intervention symptoms checker, a mood tracker, and a location tool so they can find the resources they need that are close to them, and more. This app is available for download via the Apple Store and Google play. The app consists of four key features: 1. tracker, 2. checker, 3. locater and, 4. community.

\section{1.1 Tracker}

The tracker feature includes the user-tailored validated surveys (e.g. Edinburgh Depression scales [16], GAD7 [17], PQH-9 [18]). It is designed to identify mental health difficulties and support early intervention. It provides support for users via recommendations for support and local health services (Figure 1).

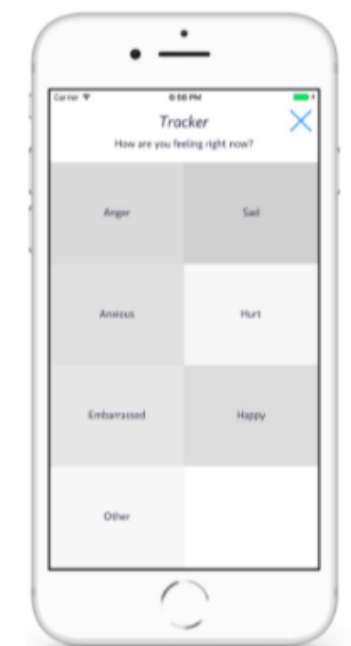

Figure 1: Moment Health App - Tracker

\subsubsection{Checker}

The checker feature is a daily mental health tracking tool. As users can input emotional data up to three times daily. Weekly reports help users to identify triggers and mental health trends. It also provides patient insight for clinicians (Figure 2).

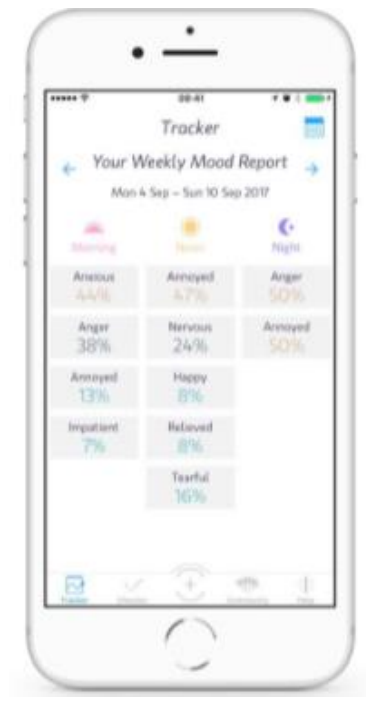

Figure 2: Moment Health App - Mood checker

\subsubsection{Locator}

The locator is a central resource of recommended healthcare centres and support groups. As the recommendations are based on users' status and geolocation (Figure 3).

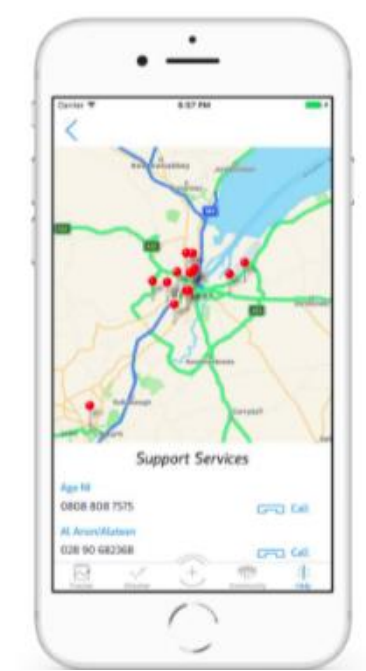

Figure 3: Moment Health App - locator

\subsubsection{Community}

The community feature consists of connectedness, support, companionship and advice from the Moment community. There is opportunity for users to add their personal network for convenient in-app referral. In addition, there is online and face to face advice and support for users seeking maternal mental health support (Figure 4). 


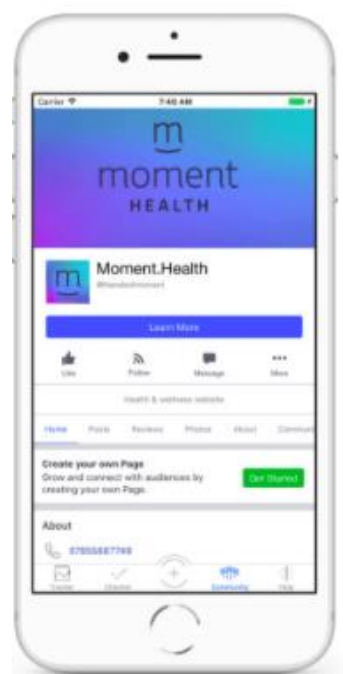

Figure 4: Moment Health App - Community

\subsection{User experience (UX) analysis}

An expert heuristic usability evaluation is where an expert uses a series of established usability and design principles to audit a user interface. This evaluation was conducted using the guiding principles, such as Jakob Nielsen's [19] 10 heuristics, principles from Gestalt psychology and the 8 golden rules by Ben Shneiderman. User experience (UX) analysis was conducted on the app, which included design observations for the different features including login screen, sign-up screen, welcome help screen, tracker, checker, locator, community, navigation design, feedback screens, help screens, and support networks. A number of recommendations were provided, which informed the development of the app, such as adding an artificial intelligence (Al) chatbot.

\section{USERS' FEEDBACK}

The app has been designed and launched in October 2017, and the premier version is due to be launched in summer 2018. To date there are 5000 users of Moment Health App and growing, with approximately 1000 active users each day. This has created a community support group for mothers, especially the Facebook group. Feedback from users' testimonies of using the Moment Health App, illustrating the positive impact on health and wellbeing are:

"I have the Moment Health app installed on my phone, as my own personal checker system. I now prioritise my mental health and ensure I dedicate a little time every day to check in with myself. l'd encourage everyone to do the same, no matter what stage of motherhood they're at". (Mother)

"My favourite feature is the Mood Tracker...I have managed to work out the times of the day when I feel most stressed and overwhelmed and now I can anticipate it and work to overcome the tension. The closed Facebook group is certainly the friendliest parenting forum I use." (Blogger)

"The app is completely free and I highly recommend it to any parent or any pregnant woman out there. This is the companion you need to help keep your mental health in check from the start of pregnancy all the way into motherhood and then all the way back around again if you so wish". (Mother)

\section{ONGOING \& FURTHER RESEARCH}

Data analytics are currently being conducted on the data generated from the app to determine the profiles and needs of the users. Further research is required to test the effectiveness of the app in improving the mental health of mothers and their families, and a clinical trial is being planned. Future work will involve adding a feature that would intelligently invoke an Al chatbot that can provide advice, coping suggestions and simulate some level of counselling at the moment of need when moods and PHQ-9 indicators indicate a progressing concern. The agreement between mood logs and PHQ-9 scores and also data mine temporal geospatial patterns of moods and anomalies that might be indicative of mood disorders or mental health issues, will also be investigated. In addition, it is anticipated to investigate how best to visualise mood logs to different users (lay person, medical professional etc.). This would give rise to affectography (perhaps a standard method to visualise mood or affect).

\section{CONCLUSION}

This programme of research has provided and is continuing to generate evidence to inform the development of the Moment Health App, to ensure that the app is evidence-based. This app is fulfilling the gap in the market for mothers to monitor with the aim to improve their mental well-being. In addition, this research is contributing to the growing area of femtech.

\section{REFERENCES}

1. World Health Organisation. (2009) Maternal mental health. Available at: http://www.who.int/maternal (Accessed 11/05/18).

2. World Health Organisation. (2017) Maternal mental health. Available at: http://www.who.int/maternal (Accessed 11/05/18). 
3. Royal College of Midwives (2017). Maternal mental health. Royal College of Midwives. UK.

4. NHS (2017). Maternal Mental Health. Available at: https://www.nhs.uk/conditions/pregnancy-andbaby/mental-health-problems-pregnant/

(Accessed 11/05/18).

5. Bauer A, Parsonage $M$, Knapp $M$, lemmi $V$, Adelaja B (2014). Costs of Perinatal Mental Health Problems. London School of Economics and Political Science, UK.

6. Royal College of Midwives (2014). Maternal mental health Improving emotional wellbeing in postnatal care. Pressure Points. Royal College of Midwives. UK.

7. Parfitt Y Ayers, S. (2012) Postnatal mental health and parenting: the importance of parental anger. Infant Mental Health Journal, 33 (4): 400410.

8. Etherington $\mathrm{N}$, McDougall J, DeWit $\mathrm{D}$, Wright $\mathrm{V}$ (2015) Maternal factors and the emotional and behavioural functioning of adolescents with chronic health conditions, Disability and Rehabilitation. Available from: https://www.researchgate.net/publication/28234797 2 Maternal Factors and the Emotional and Beh avioural Outcomes of Adolescents with Chronic Health Conditions (Accessed 11/05/18).

9. Royal College of Obstetricians and Gynaecologists (RCOG). (2017) Maternal mental health - women's voices Available at: https://www.rcog.org.uk/globalassets/documents/pa tients/information/maternalmental-healthwomensvoices.pdf (Accessed 11/05/18).

10. Maternal Mental Health Alliance. (2017) Maternal mental health. Available at: https://maternalmentalhealthalliance.org (Accessed 11/05/18).

11. NICE (2016) NICE Quality Standard Antenatal and postnatal mental health. Available at: https://www.nice.org.uk/guidance/qs115/resour ces/antenatal-and-postnatal-mental-health-pdf75545299789765 (Accessed 11/05/18).

12. Cheug LC. (2017). Femtech: the 'niche' sector pregnant with possibilities. Available at: https://medtechengine.com/article/femtech/ (Accessed 11/05/18).

13. Caroll J, Moorhead A, Bond R, LeBlanc W, Petrella R, Fiscella K. (2017) Who Uses Mobile Phone Health Apps and Does Use Matter? A Secondary Data Analytics Approach. Journal of Medical Internet Research, 19 (4) e125.
14. Moorhead A, Hazlett D, Harrison L, Carroll J, Irwin, A, Hoving C. (2013) A New Dimension of Health Care: Systematic Review of the Uses, Benefits, and Limitations of Social Media for Health Communication. Journal of Medical Internet Research, 15 (4). e85.

15. O'Neill S, Armour C, Bolton D, Bunting B, Corry C, Devine B, Ennis E, Ferry F, McJenna A, Mclafferty M. Murphy, S. (2015) Towards a better future: The transgenerational impact of the Troubles on Mental Health. CVSNI, Belfast.

16. Cox JL, Holden JM, Sagovsky R. (1987) Detection of postnatal depression: Development of the 10-item Edinburgh Postnatal Depression Scale. British Journal of Psychiatry, 150:782-786.

17. Kroenke K, Spitxer RL, Williams JB. (2001) The PHQ-9: validity of a brief depression severity measure. Journal of General Internal Medicine, 16(9):606-13.

18. Goldberg D. (1978) Manual of the General Health Questionnaire. NFER-Nelson, Windsor.

19. Nielsen J. (1995) 10 Usability Heuristics for User Interface Design. Avaailbe at:

https://www.nngroup.com/articles/ten-usabilityheuristics/ (Accessed 11/05/18). 